\title{
INTEGRATION OF SMAP AND SMOS L-BAND OBSERVATIONS
}

\author{
Rajat Bindlish ${ }^{1}$, Thomas J. Jackson ${ }^{2}$, Steven Chan ${ }^{3}$, Andreas Colliander ${ }^{3}$, Yann Kerr ${ }^{4}$ \\ ${ }^{1}$ NASA Goddard Space Flight Center, Greenbelt, MD, 20771, USA \\ email: rajat.bindlish@nasa.gov \\ ${ }^{2}$ USDA ARS Hydrology and Remote Sensing Laboratory, Beltsville, MD \\ ${ }^{3}$ Jet Propulsion Lab, Pasadena, CA \\ ${ }^{4}$ CESBIO, France
}

\begin{abstract}
Soil Moisture Active Passive (SMAP) mission and the ESA Soil Moisture and Ocean Salinity (SMOS) missions provide brightness temperature and soil moisture estimates every 2-3 days. SMAP brightness temperature observations were compared with SMOS observations at $40^{\circ}$ incidence angle. The brightness temperatures from the two missions are not consistent and have a bias of about $2.7 \mathrm{~K}$ over land with respect to each other. SMAP and SMOS missions use different retrieval algorithms and ancillary datasets which result in further inconsistencies between the soil moisture products. The reprocessed constant-angle SMOS brightness temperatures were used in the SMAP soil moisture retrieval algorithm to develop a consistent multi-satellite product. The integrated product will have an increased global revisit frequency (1 day) and period of record that would be unattainable by either one of the satellites alone. Results from the development and validation of the integrated product will be presented.
\end{abstract}

Index Terms - SMAP, SMOS, passive microwave, inter-comparison of microwave radiometers

\section{INTRODUCTION}

Soil moisture observations from the Soil Moisture Active Passive (SMAP) mission [1] and the ESA Soil Moisture and Ocean Salinity (SMOS) missions [2] provide information about an important hydrologic parameter that contributes to understanding the Earth's climate and water cycles. The standard SMOS and SMAP soil moisture products currently have a revisit frequency of about 3 days. The value and range of applications for the SMAP soil moisture product is dependent on the revisit frequency of the soil moisture observations. Integration of all available (both AM and PM) brightness temperature (TB) observations from multiple Lband satellites (SMAP and SMOS) can potentially reduce the revisit time to about 1 day.
The SMOS and SMAP missions use different algorithms and ancillary datasets to estimate soil moisture, the choices are dependent on the instrument configuration. The SMOS soil moisture algorithm exploits its multi-angle observations [2]. This algorithm cannot be applied to SMAP TB observations that are acquired at a fixed incidence angle. Moreover, there are several differences in the ancillary data sources (for example: SMAP uses GMAO GEOS-5 model estimates for surface temperature and SMOS uses ECMWF surface temperature estimates). These differences can result in discrepancies in the soil moisture retrievals between the two products. As a result it is not possible to develop a consistent soil moisture climate data record by just merging the soil moisture products from the two missions.

The first step in the development of the integrated product requires that the TBs from the two missions are consistent with each other. A physically-based retrieval algorithm that spans multiple L-band missions requires consistent input observations for the development of a long term environmental data record. Availability of consistent TB observations from SMOS and SMAP satellites will allow the development of a consistent long term soil moisture data record.

Consistent calibration across both SMOS and SMAP satellite missions is critical to developing a long term climate data record of L-band TB observations. SMOS TB observations were reprocessed to develop a fixed $40^{\circ}$ incidence angle product (consistent with the SMAP Level 1 radiometer observations) (referred as the SMOS-SMAP TB product). A physically-based soil moisture algorithm that spans multiple L-band missions requires consistent input observations for the development of a long term environmental data record. SMOS-SMAP TB observations will then be used in the SMAP radiometer only soil moisture retrieval algorithm with SMAP ancillary data to develop a consistent soil moisture product. This results in the development of a harmonized soil moisture product using the same soil moisture retrieval algorithm. 


\section{BRIGHTNESS TEMPERATURE INTER- COMPARISON METHODOLOGY}

Microwave observations from the SMOS mission were reprocessed to approximate SMAP microwave radiometer observations made at a constant incidence angle of $40.0^{\circ}$. Only the alias-free portions of the SMOS field-of-view were used in the comparison. Additionally, the alias-free portions of the swath provide brightness temperatures with the lowest $\mathrm{NE} \Delta \mathrm{T}$ [3]. SMOS data version v620 was used for the analysis.

L-band observations are a function of land surface conditions (e.g., soil moisture, surface temperature, vegetation), which vary both in space and time. Although vegetation conditions do not rapidly change in time, soil moisture and soil temperature can vary significantly over a short period. In order to minimize inter-comparison errors associated with temporal changes in soil moisture and temperature, a maximum time window between the two satellite observations of $30 \mathrm{~min}$ was used. Both SMAP and SMOS have an average $3-\mathrm{dB}$ footprint size of $40 \mathrm{~km}$. Spatial variations in the contributing area were minimized by only using observations when the footprint distance was less than $1 \mathrm{~km}$ between SMAP and SMOS. Brightness temperatures at the top of the atmosphere (TOA) from both missions were used in the inter-comparison. Comparisons were made with brightness temperature without reflected galaxy correction, ionosphere or atmospheric correction. RFI flags from both the missions were used in the analysis. Only brightness temperature observations when both the missions indicated no significant RFI were used in the match-up analysis. The azimuth angle of the observations was ignored during the analysis. This analysis was done for both the horizontal $(\mathrm{H})$ and vertical $(\mathrm{V})$ polarizations.

\section{BRIGHTNESS TEMPERATURE INTER- COMPARISON RESULTS}

Figure 1 (a-b) shows the density plot of the brightness temperature (top of the atmosphere) comparison between SMOS and SMAP over land targets $40^{\circ}$ incidence angle for $\mathrm{V}$ - and H-polarizations. The current L1B radiometer data (R13080) were compared with the most recent SMOS L1B data (version 620) for this analysis. Statistical analysis results are summarized in Table 1. The SMAP brightness temperatures show a very strong correlation with the SMOS observations and most of the observations fall along the 1:1 line. The scatter is greater for $\mathrm{H}$ polarization observations, which are more sensitive to changes in land surface conditions (soil moisture and surface temperature). Some of the scatter in the inter-comparison is likely due to the presence of RFI in either or both of the SMAP or SMOS observations. Land surface heterogeneity of the footprint can also result in some scatter.

SMAP observations show a colder TB bias (about $2.7 \mathrm{~K}$ ) as compared to SMOS for both polarizations. Most of the RMSD can be attributed to the bias between the two satellites. Global average brightness temperature comparisons over ocean areas with SMOS are quite favorable indicating less than $0.4 \mathrm{~K}$ mean bias at top of the atmosphere.

In addition, we extracted the equivalent data set over oceans. These combined results provide strong evidence of the relative calibration of SMAP and SMOS over a wide range of targets. The SMAP brightness temperature compared well with SMOS observations over oceans. The observations over the ocean target have a small dynamic range $(5 \mathrm{~K})$ but lie along the $1: 1$ line with no significant bias. The correlation coefficient for just the ocean observations is due to the small dynamic range.

A future but small change in reflector or radome emissivity (predicted for the next major TB data release, likely in 2017) will subtly modify this bias [4]. Efforts will be made to address these differences in TB calibration over land and to develop a consistent L-band brightness temperature dataset between SMOS and SMAP missions. A linear adjustment over land was made to recalibrate the SMAP brightness temperatures to the SMOS calibration to develop a consistent brightness temperature data record.

\section{CONSISTENT L-BAND DATA PRODUCT}

SMOS and SMAP both have the same local overpass time of 6 AM/PM. The SMOS and SMAP orbits are opposite to each other (one will be ascending when the other is descending) and the two satellites cross each other at the equator at $6 \mathrm{AM}$ and $6 \mathrm{PM}$ (SMAP is $6 \mathrm{AM}$ descending orbit whereas SMOS is $6 \mathrm{AM}$ ascending orbit). SMAP has a swath width of about $1000 \mathrm{~km}$. SMOS also has a swath width of about $1000 \mathrm{~km}$.

The SMAP revisit time is about 3 days (using descending orbits only). Figure $2 \mathrm{a}$ shows the global coverage of SMAP TB for a single day using both ascending and descending orbits. Even using both the SMAP ascending and descending orbits there are still significant gaps in the global coverage for a single day of observations. Figure $2 b$ shows the global coverage of TB using both SMAP and SMOS satellites for both ascending and descending orbits. The addition of both SMAP and SMOS observations greatly increases the spatial coverage for a single day. The use of both satellites and both ascending and descending orbits results in near complete global coverage within a single day. Moreover, large portions of the globe would have coverage at both $6 \mathrm{AM}$ and $6 \mathrm{PM}$ local time.

SMOS-SMAP TB observations will then be used in the SMAP radiometer only soil moisture retrieval algorithm with SMAP ancillary data to develop a consistent soil moisture product. This resulted in the development of a harmonized soil moisture product using the same soil moisture retrieval algorithm.

The soil moisture retrievals using SMOS-SMAP TB observations will be compared directly with SMOS and 
SMAP only retrievals. The integrated soil moisture product will be also validated with the same set of core and candidate validation in situ observations used for the standard L2SMP product. Results from the validation analysis will be presented. This work will help in the development of a consistent multi-satellite soil moisture product using observations from SMOS and SMAP missions.

\section{REFERENCES}

[1] S. Chan, R. Bindlish, P. O’Neill, E. Njoku, T. Jackson, A. Colliander, F. Chen, M. Mariko, S. Dunbar, J. Piepmeier, S. Yueh, D. Entekhabi, M. Cosh, T. Caldwell, J. Walker, X. Wu, A. Berg, T. Rowlandson, A. Pacheco, H. McNairn, M. Thibeault, J. MartinezFernandez, A. Gonzalez-Zamora, M. Seyfried, D. Bosch, P. Starks, D. Goodrich, J. Prueger, M. Palecki, E. Small, M. Zreda, J. Calvet, W. Crow, Y. Kerr: Assessment of the SMAP passive soil moisture product, IEEE Transactions on Geoscience and Remote Sensing, 54(8), pp. 4994-5007, Aug 2016.
[2] Y.H. Kerr, P. Waldteufel, J.P. Wigneron, S. Delwart, F. Cabot, J. Boutin, M.J. Escorihuela, J. Font, N. Reul, C. Gruhier, S.E. Juglea, M.R. Drinkwater, A. Hahne, M. Martin-Neira, S. Mecklenburg, "The SMOS Mission: New Tool for Monitoring Key Elements ofthe Global Water Cycle," Proceedings of the IEEE , vol.98, no.5, pp.666-687, 2010.

[3] R. Oliva, M. M. Neira, I. Corbella, F. Torres, J. Kainulainen, J. E. Tenerelli,, F. Cabot, and F. M. Porqueras "SMOS Calibration and Instrument Performance after One Year in Orbit," IEEE Trans. Geosci. Remote Sens., vol. 51, no. 1, pp. 654-670, Jan 2013.

[4] J. R. Piepmeier and S. Chan, "SMAP Radiometer Brightness Temperature Calibration for the L1B_TB and L1C_TB Validated Version 3 Data Products," Jet Propulsion Laboratory, California Institute of Technology, Pasadena, CA, JPL D-93718, 2016. https://nsidc.org/sites/nsidc.org/files/files/SMAP\%2520L1B_TB\% 2520Validated $\% 2520$ Release $\% 2520$ Assessment $\% 2520$ Report $\% 25$ 20FINAL\%5B1\%5D.pdf.
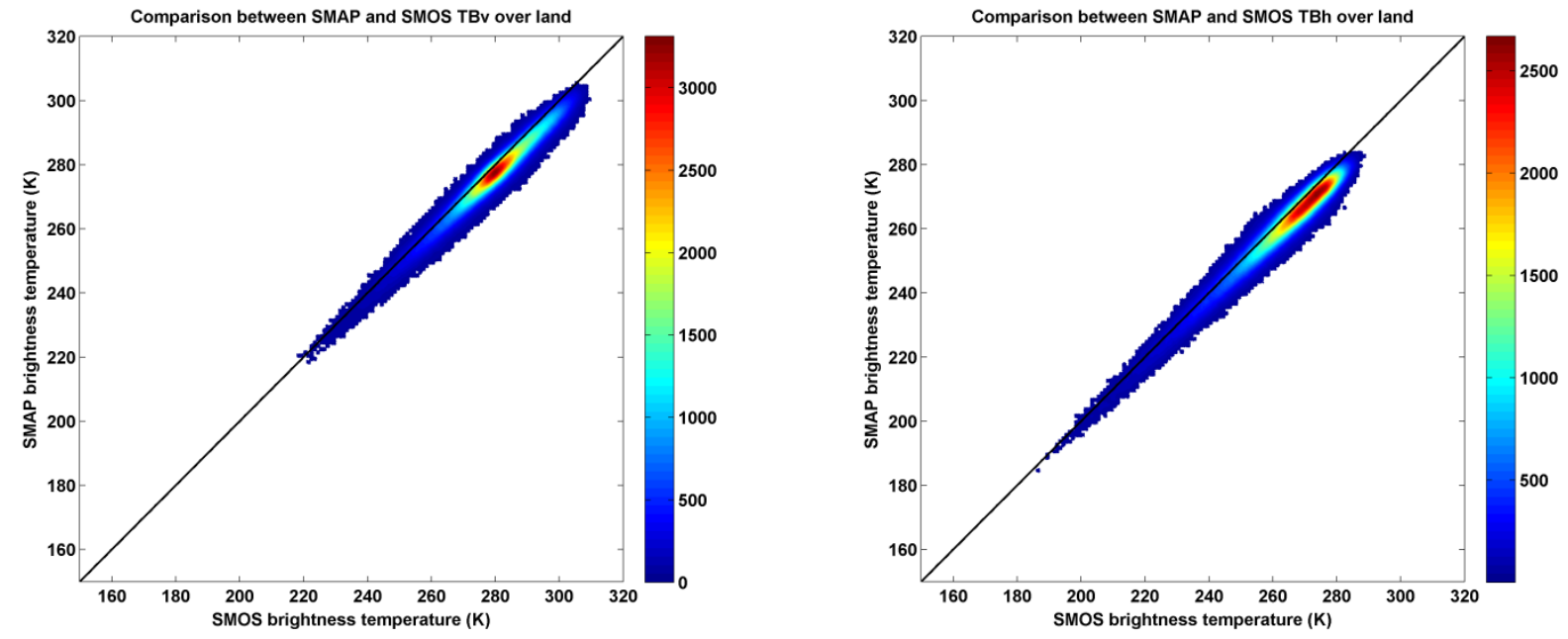

Figure 3.1 Density plot of the L1 brightness temperature comparison (top of the atmosphere) between SMAP (R13080) and SMOS (version 620) observations over land targets for V-pol (left) and H-pol (right).

Table 1. Summary statistics of the brightness temperature comparison between SMOS (version 620) and SMAP (R13080) for May 5, 2015-October 31, 2016.

\begin{tabular}{|c|c|c|c|c|c|}
\hline & & RMSD (K) & $\mathbf{R}$ & Bias [SMAP-SMOS] (K) & ubRMSD (K) \\
\hline \multirow{4}{*}{ H pol } & Land & 4.34 & 0.9775 & -2.65 & 3.44 \\
\cline { 2 - 6 } & Ocean & 2.45 & 0.7061 & 0.08 & 2.45 \\
\cline { 2 - 6 } & Overall & 2.92 & 0.9994 & -0.60 & 2.86 \\
\hline \multirow{4}{*}{ V pol } & Land & 4.21 & 0.9745 & -2.71 & 3.22 \\
\cline { 2 - 6 } & Ocean & 2.57 & 0.7679 & 0.57 & 2.51 \\
\cline { 2 - 6 } & Overall & 2.98 & 0.9994 & -0.25 & 2.97 \\
\hline
\end{tabular}



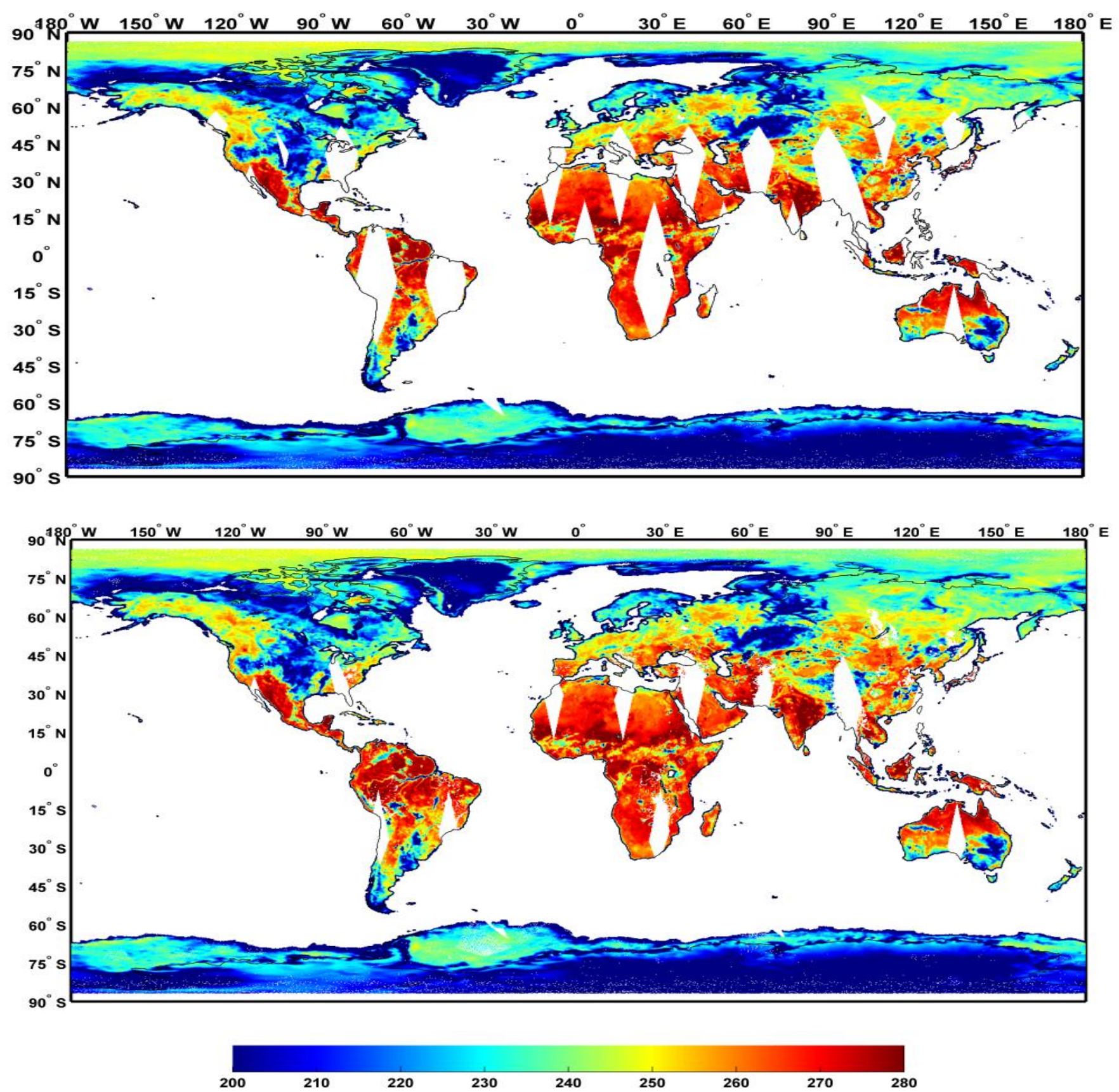

Figure 2: Global coverage of (a) SMAP TB using both ascending and descending orbits, and (b) SMAP and SMOS TB using both ascending and descending orbits for May 21, 2015. The figures show the added value of using both satellites and both ascending and descending orbits. 